\title{
Synthesis of One Dimensional Electromagnetic Bandgap Structures with Fully Controlled Parameters
}

\author{
Israel Arnedo, Member, IEEE, Magdalena Chudzik, Jon M. Percaz, Ivan Arregui, Member, IEEE, \\ Fernando Teberio, Member, IEEE, David Benito, Txema Lopetegi, Member, IEEE, and \\ Miguel A. G. Laso, Senior Member, IEEE
}

\begin{abstract}
In this paper, we propose a novel synthesis strategy for the design of One Dimensional Electromagnetic Bandgap (1DEBG) structures where all the performance parameters of these devices can be fully controlled, i.e., the central frequency of the forbidden band, its attenuation level and bandwidth, and the ripple level at the passbands. The novel synthesis strategy employs a new inverse scattering technique to accurately synthesize the 1DEBG structure, targeting a properly interpolated version of a classical periodic filter fulfilling the required frequency specifications. The new inverse scattering technique follows a continuous layer peeling approach and relies on the coupled-mode theory to precisely model the microwave structures. Telecommunication and radar systems, as well as material characterization devices, will be profited by this proposal with which enhanced filters, sensors, power dividers, couplers, mixers, oscillators and amplifiers can be designed in many different technologies. As a proof of concept, a 1D-EBG structure in microstrip technology with a single forbidden band (free of spurious stopband replicas), with attenuation level of $30 \mathrm{~dB}$, fractional bandwidth larger than $100 \%$, and return loss level at the passbands of $20 \mathrm{~dB}$, has been designed and fabricated. The measurements obtained are in very good agreement with the simulations and target specifications, being free of spurious replicas up to the 15 th harmonic, showing the robustness and very good performance of the novel design strategy proposed.
\end{abstract}

Index Terms - electromagnetic bandgap, synthesis, inverse problems, coupled-mode theory, microstrip, filters.

\section{INTRODUCTION}

$\mathrm{E}$ LECTROMAGNETIC bandgap (EBG) structures are periodic or quasi-periodic structures featuring variations of the electromagnetic properties of the medium, originally designed in an intuitive manner gathered from quantum mechanics and solid-state physics. In a nutshell, analogously to how in a periodic crystal lattice formed by atoms and molecules there may be gaps in the energy band structure of the crystal where the electrons are forbidden within certain energies and directions, in a EBG structure there may be frequency bands at which electromagnetic waves are not able to propagate [1]-[3]. This novel idea was originally introduced by Yablonovitch [4] and John [5] simultaneously in the latest 1980s, at optical

Manuscript received Jan. 15, 2017. This work was supported by MINECO (Spain) under Projects TEC2014-51902-C2-2-R and TEC2014-55735-C3-R. This paper is an expanded version from the IEEE MTT-S Latin America Microwave Conference, Dec. 12-14, 2016, Puerto Vallarta, Mexico. frequencies. Apart from the thriving applications of 3D- and 2D-EBGs in antennas and radiation structures [6]-[9], 1D-EBG structures lead to the design of many devices with enhanced performance in a wide variety of technologies such as microstrip [10], perturbed ground-plane microstrip [11], coplanar lines [12], rectangular waveguide [13]-[15], trough waveguide [16], circular waveguide [17], SIW (Substrate Integrated Waveguide) and HMSIW (Half-Mode SIW) [18][20], and finline waveguide [21], among others.

In microstrip technology, the concept was successfully introduced by Itoh in the latest 90s [22]. Since then, a great effort has been done to accurately design devices with performances highly competitive in many different applications such as telecommunications, radar and sensors. Of particular interest has been the use of 1D-EBGs for broadband harmonic tuning in power amplifiers [23], [24] and oscillators [25], [26] to increase the amplifier output power and efficiency, and in mixers [27], [28] to reduce noise. Very good results have been obtained as well with the use of 1D-EBGs for increasing the coupling level and isolation in couplers [29], [30], compacting power dividers [31], and enhancing the sensitivity of sensors [32]-[34].

The use of EBG structures for the design of microwave, millimeter-wave and terahertz filters is of special significance. Very competitive devices have been obtained in microstrip lowpass filters performing high rejection level and/or wide rejected band width [35]-[38], and in microstrip and coplanar waveguide band-pass filters with spurious suppression and/or compact layout [39]-[45]. Some of the latest use periodic capacitive loaded and/or inductively loaded lines exhibiting slow-wave behavior and featuring spurious suppression and reduced size. Finally, 1D-EBG microstrip structures were since the beginning of great importance for the design of reject-band filters [46], [47]. Recently, reject-band filters with tunable capabilities [48] and reject-band filters for broadband rejection of the commonmode of the differential lines [49]-[51] have been successfully proposed.

Many different design procedures have been followed to control the relevant parameters of the EBG for the intended application [3], [52]. The central frequency of the stopband is

The authors are with the Electrical and Electronic Engineering Department, Public University of Navarre, 31006 Pamplona, Spain. E-mail: israel.arnedo@unavarra.es 
easily ruled by the period, and the rejection level and bandwidth are controlled by the shape of the pattern, arrangement, location and its size [53], [19]. Tapering the size and locations of those periodic patterns has been the usual strategy to obtain a progressive matching between the periodic structure Blochwaves and the characteristic impedance at the ports, reducing the ripple out of the forbidden band [54]. Two papers covering an alternative strategy for the design of 1D-EBGs must be highlighted in this respect. First, in [55] a filter is designed modeling accurately the unit cell of the EBG with $L s$ and $C s$, obtaining very good results for a low-pass response. Secondly, in [56] a reject-band filter is designed using the $A B C D$ matrix to model the unit cell as two transmission line sections with a series (parallel) inductor (capacitor) in between; a very good result is also obtained for the rejection band but spurious harmonic bands appear. Eventually, the difficulty of the task to obtain very good results has yielded even to the use of genetic algorithms [57]-[59].

In this paper, we extend the work presented in [60], where several techniques to improve the performance and design flexibility of 1D-EBG structures were reviewed, together with some very interesting applications. A systematic design procedure for EBG structures with fully controlled parameters was still not available, though. Here we present a new and general 1D-EBG design methodology able to accomplish periodic and quasi-periodic EBG structures with fully controlled design parameters such as the passband ripple level, stopband rejection level and bandwidth, and with no spurious stopbands. To achieve this goal, the coupled-mode formulation and inverse scattering synthesis procedures will be employed. In Section II, the novel design methodology proposed will be rigorously presented and, in Section III, a 1D-EBG structure will be designed and successfully demonstrated in microstrip technology as a proof of concept. A comparison of its performance with respect to the results found in the literature will be also provided. Finally, Section IV summarizes our findings and concludes the paper.

\section{SyNTHESIS METHODOLOGY}

The novel synthesis methodology to design 1D-EBG structures with no spurious stopbands and all the parameters under control will be explained in detail in this Section.

\section{A. Spurious-free $1 D-E B G$}

As it is detailed in [61] and [62], using the coupled-mode theory [63] and assuming single-mode operation, the electromagnetic behavior of a 1D-EBG structure implemented in microwave technology can be characterized through the coupling coefficient, $K(z)$, between the forward and backward travelling waves associated to the operation mode, and the simplified system of coupled-mode equations:

$$
\begin{aligned}
& \frac{d a^{+}}{d z}=-j \cdot \beta \cdot a^{+}+K \cdot a^{-} \\
& \frac{d a^{-}}{d z}=j \cdot \beta \cdot a^{-}+K \cdot a^{+}
\end{aligned}
$$

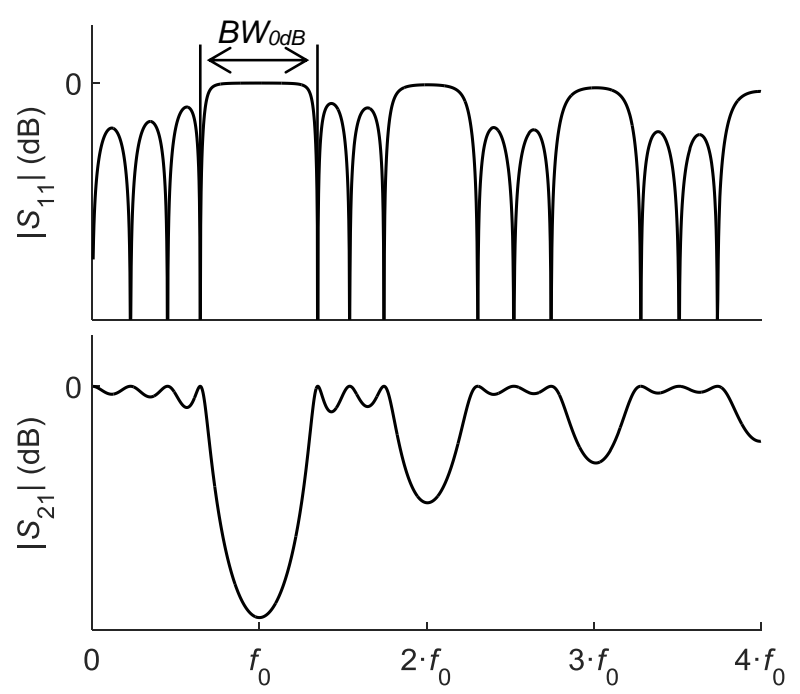

Fig. 1. Scheme of the frequency response of a generic 1D-EBG

where:

$$
\begin{aligned}
& \widehat{\vec{E}}=a^{+} \cdot \vec{E}^{+}+a^{-} \cdot \vec{E}^{-} \\
& \widehat{\vec{H}}=a^{+} \cdot \vec{H}^{+}+a^{-} \cdot \vec{H}^{-}
\end{aligned}
$$

being $\widehat{\vec{E}}, \widehat{\vec{H}}$ the total electric and magnetic fields present in the structure; $\vec{E}^{+}, \vec{H}^{+}, \vec{E}^{-}, \vec{H}^{-}$the vector mode patterns of the forward (+) and backward (-) traveling waves corresponding to the operation mode in the auxiliary uniform waveguide associated to the cross-section of interest; $\beta$ the phase constant of the mode; $z$ the direction of propagation; and $a^{+}, a^{-}$, the complex amplitudes of the forward (+) and backward (-) traveling waves along the non-uniform waveguide.

If the 1D-EBG structure is periodic along the propagation axis $z$ with period $\Lambda$, then the $K(z)$ produced by the perturbation is also periodic with the same period and, hence, it can be expanded in a Fourier series as:

$$
\begin{gathered}
K(z)=\sum_{n=-\infty}^{n=\infty} K_{n} \cdot e^{j \cdot \frac{2 \cdot \pi}{\Lambda} \cdot n \cdot z} \\
K_{n}=\frac{1}{\Lambda} \cdot \int_{\Lambda} K(z) \cdot e^{-j \cdot \frac{2 \cdot \pi}{\Lambda} \cdot n \cdot z} \cdot d z
\end{gathered}
$$

As it is explained in [64], each forbidden band of the 1DEBG is linked to the corresponding $K_{n}$, see Fig. 1. If a spuriousfree 1D-EBG structure is to be designed, a sinusoidal coupling coefficient must be taken (because it is the function that makes all $K_{n}$ equal to zero except for $K_{ \pm 1}$ ) with the following design equations given in terms of the central frequency of the forbidden band, $f_{0}$, its rejection level, $\left|S_{21}\right|_{\min }$, and its bandwidth defined between rejection zeros, $B W_{0 d B}$ :

$$
\Lambda=\frac{c}{2 \cdot f_{0} \cdot \sqrt{\varepsilon_{e f f}}}
$$




$$
\begin{gathered}
A=\frac{2 \pi \cdot B W_{0 d B} \cdot \sqrt{\varepsilon_{e f f}} \cdot \operatorname{sech}^{-1}\left(\left|S_{21}\right|_{\text {min }}\right)}{c \cdot \sqrt{\left[\operatorname{sech}^{-1}\left(\left|S_{21}\right|_{\text {min }}\right)\right]^{2}+\pi^{2}}} \\
L=\frac{c \cdot \sqrt{\left[\operatorname{sech}^{-1}\left(\left|S_{21}\right|_{\text {min }}\right)\right]^{2}+\pi^{2}}}{\pi \cdot B W_{0 d B} \cdot \sqrt{\varepsilon_{e f f}}}
\end{gathered}
$$

where $c$ is the speed of light in vacuum, $A$ is the amplitude of the sinusoidal coupling coefficient, $L$ is the device length, and $\varepsilon_{e f f}$ is the mean value of $\varepsilon_{e f f}(z)$ in a period, calculated as $\varepsilon_{e f f}=\left(1 / \Lambda \cdot \int_{\Lambda} \sqrt{\varepsilon_{e f f}(z)} \cdot d z\right)^{2}$.

Unfortunately, this analytical design procedure does not provide any control over the ripple level in the passbands. Many efforts have been carried out using tapering functions (smooth variations of the amplitude or the size of the perturbation in the propagation direction) [36], [54], along with period alterations (variations of the periodicity along the propagation direction) [65], to reduce the ripple level out of the forbidden band by means of a non-controlled progressive matching between the periodic structure Bloch-waves and the characteristic impedance at the ports. Both strategies increase the degrees of freedom that can be used by optimization routines and they achieve good results [19], [53]. However both modifications break the pure periodicity of the 1D-EBG structure and, consequently, the design equations become inaccurate or even invalid if an extreme case is applied. Unlike in these previous approaches, a direct synthesis strategy is proposed in this paper. Our technique is able to achieve the desired ripple level (while keeping full control over the rest of the design parameters) using classical filter theory of periodic structures and an inverse scattering synthesis method.

\section{B. Classical Filter Theory of Periodic Structures}

Classical filter theory provides expressions to design filters using periodic structures. The idea rests on cascading $N$ transmission line sections with different characteristic impedances and the same electrical length [66], [67]. This electrical length corresponds to $\pi / 2$ at the frequency of maximum rejection, $f_{0}$, where the maximum attenuation level, $\left|S_{21}\right|_{\text {min }}$, is achieved. The resulting devices are, strictly speaking, quasi-periodic structures with constant perturbation period and carefully "tapered" characteristic impedance profile to achieve the required filtering function. The method inherently provides spurious replicas of the designed stopband at $3 \cdot f_{0}, 5 \cdot f_{0}, \ldots$, which correspond to $\pi$-increments in the electrical length, i.e., $3 \pi / 2,5 \pi / 2, \ldots$, respectively.

For the case of the Chebyshev function, the rejection bandwidth $(B W)$ and return loss level out of stopband ( $R L$ in $\mathrm{dB})$ will be also specified, see Fig. 2, and transformed into the $\alpha$ and $\eta$ parameters:

$$
\alpha=\cos \left(\frac{\pi}{4} \cdot \frac{B W}{f_{0}}\right)
$$

$$
\eta=\sinh \left[\frac{1}{N} \cdot \sinh ^{-1}\left(\sqrt{10^{\frac{R L}{10}}-1}\right)\right]
$$

The order of the Chebyshev filter, $N$, necessary to satisfy the requirements can be estimated by means of:

$$
N=\frac{\cosh ^{-1}\left(\sqrt{\left(\frac{1}{\left|S_{21}\right|_{\text {min }}^{2}}-1\right) \cdot\left(10^{\frac{R L}{10}}-1\right)}\right)}{\operatorname{sech}^{-1}(\alpha)}
$$

A general procedure to obtain the approximated values for the characteristic impedances of the consecutive transmission line sections of the Chebyshev periodic filter, $Z_{i}$, is the following [67]:

$$
\begin{array}{ll}
Z_{i}=\frac{1}{Z_{i}^{\prime}} \cdot Z_{0} & \text { for } i \text { odd } \\
Z_{i}=Z_{i}^{\prime} \cdot Z_{0} & \text { for } i \text { even }
\end{array}
$$

where $i$ is an integer number from 0 to $N$, being $N$ the order of the filter (and the number of sections), $Z_{0}$ is the characteristic impedance of the system input port, and assuming thus $Z_{0}^{\prime}=1$. Next, for $i=1, \ldots, N$ the element values of the filter can be calculated using the following approximate expressions [67]:

$$
\begin{gathered}
Z_{i}^{\prime}=B_{i} \cdot\left(\frac{2 \cdot \sin \left[(2 \cdot i-1) \cdot \frac{\pi}{2 \cdot N}\right]}{\alpha}-\frac{\alpha}{4}\right. \\
\left.\cdot\left\{\frac{\eta^{2}+\sin ^{2}\left(\frac{i \cdot \pi}{N}\right)}{\sin \left[(2 \cdot i+1) \cdot \frac{\pi}{2 \cdot N}\right]}+\frac{\eta^{2}+\sin ^{2}\left[\frac{(i-1) \cdot \pi}{N}\right]}{\sin \left[(2 \cdot i-3) \cdot \frac{\pi}{2 \cdot N}\right]}\right\}\right)
\end{gathered}
$$

with

$$
B_{i}=\frac{\left\{\eta^{2}+\sin ^{2}\left[\frac{(i-2) \cdot \pi}{N}\right]\right\}\left\{\eta^{2}+\sin ^{2}\left[\frac{(i-4) \cdot \pi}{N}\right]\right\} \cdots}{\left\{\eta^{2}+\sin ^{2}\left[\frac{(i-1) \cdot \pi}{N}\right]\right\}\left\{\eta^{2}+\sin ^{2}\left[\frac{(i-3) \cdot \pi}{N}\right]\right\} \cdots}
$$

where the last term $\eta^{2}+\sin ^{2}[0]$, that could appear in the numerator or denominator, is replaced by $\eta$. Finally, the impedance of the output port, $Z_{N+1}$, is equal to:

$$
\begin{array}{cc}
Z_{N+1}=Z_{0} & \text { for } N \text { odd } \\
Z_{N+1}=\frac{10^{\frac{R L}{20}}-1}{10^{\frac{R L}{20}}+1} \cdot Z_{0} & \text { for } N \text { even }
\end{array}
$$

When higher precision for the values of the characteristic impedances of the filter is required, the numerical solution provided by Keysight ${ }^{\mathrm{TM}}$ Genesys S/Filter software tool will be employed. 


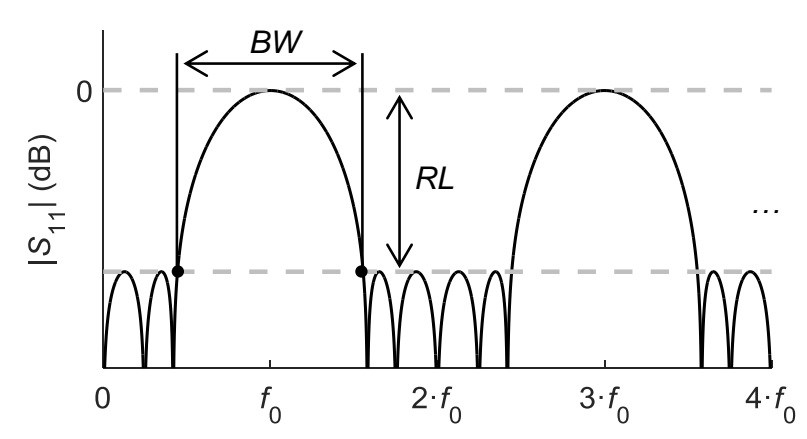

Fig. 2. Scheme of the frequency response of a generic $N$ th-section classical Chebyshev periodic filter.

A final remark must be done about the length of the transmission line sections of the classical periodic filters within the context of the EBG notation. Indeed, it is important to note that the periodicity $\Lambda$ obtained in (4) for $1 \mathrm{D}-\mathrm{EBG}$, is related to the same frequency $f_{0}$ defined in this subsection for classical periodic filters (in both cases $f_{0}$ is the frequency of maximum rejection). Thus, the electrical length of the period $\Lambda$ of the 1DEBG at $f_{0}$ obtained using (4) is $\pi$. Here, the electrical length of the transmission line section is $\pi / 2$ at $f_{0}$. This means that we can consider that two transmission line sections of the classical periodic filter conform a period, so to speak, of the 1D-EBG or, in other words, the transmission line sections of the classical periodic filter theory have a length of $\Lambda / 2$, which is fully consistent.

\section{New Inverse Scattering Synthesis Method: Continuous Layer Peeling}

In order to synthesize the spurious-free 1D-EBG structure with full control over all the design parameters, the frequency response of the classical periodic filter obtained in the previous subsection is computed (by the transfer matrix multiplication of the ideal transmission line sections) as a starting point. Then, that periodic frequency response is modified by simply removing all the spectral content above $2 \cdot f_{0}$, as it can be seen in Fig. 3. In this way, we obtain the target frequency response for our 1D-EBG structure that fulfills all the design requirements (central frequency, bandwidth and rejection level for the stopband, and return loss level for the passbands), being simultaneously free of spurious stopbands. The use of the frequency response of the classical periodic filter as starting point guarantees that the length of the 1D-EBG device is fully controlled (similar to the length of the classical periodic filter and controlled by the filter order). In the same way, the characteristic impedance excursion of the 1D-EBG device will be also kept under control (similar to the excursion of the classical periodic filter).

Now, a novel synthesis method based on inverse scattering is applied to the target frequency response obtained, to recover the desired 1D-EBG structure. In the past, we proposed a general inverse scattering synthesis method, given as a series solution of the synthesis problem, valid for the design of matched filters [62], pulse shapers [68] or differentiators [69], among other devices. As we reported, an exceedingly high number of terms

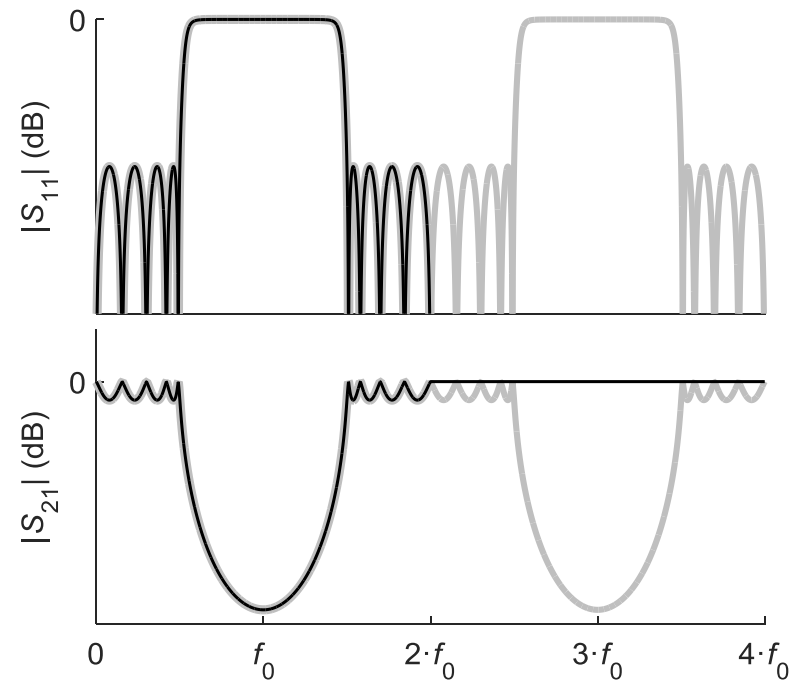

Fig. 3. Scheme of the frequency response of a generic $N$ th-section classical Chebyshev periodic filter (grey line) and its transformation to the target frequency response used in the continuous layer peeling synthesis method (black line).

in the series solution are needed in order to synthesize devices with high reflectivity [70], making the method unpractical for those high reflectivity cases. An alternative inverse scattering synthesis method was presented for devices demanding high reflectivity [71], but the method is limited to rational frequency responses, being not valid for the target frequency responses of our 1D-EBG structures, which are defined numerically and require a high rejection level. Therefore, a new inverse scattering synthesis method will be proposed and employed in this paper, surpassing the limitations of the two previous methods, and adequate for our 1D-EBG structures. Inspired by the work of Feced et al. in [72] for non-uniform Fiber Bragg Gratings in the optics field, a continuous layer peeling synthesis method will be derived here for the microwave field. The layer peeling algorithms arise directly from the idea of causality [73]. Assuming that in $t=0$ a one dimensional device is excited by an ideal impulse, the response of the device in reflection at $t=$ $\Delta t$ must be only determined by the first layer of the device of length $\Delta z$, provided that $\Delta t$ is the time required for the wave to go forward and back that first layer of length $\Delta z$. The modelling of the device will be done using the coupled-mode theory formalism, and it will be implemented in the frequency domain where it is more computationally efficient [74]. Thus, the synthesis algorithm will consist of three steps that will be iterated along the device: a) the coupling coefficient at $z=0$, $K(0)$, is derived from the target frequency response in reflection, b) the target frequency response is propagated through the device one infinitesimal layer using the Riccati equation with the value just calculated $K(0)$, c) the propagated frequency response becomes the new target response, effectively peeling off the obtained layer and allowing us to calculate the next value for $K(z)$ by returning again to step a).

As it was demonstrated in [62], there exits an analytical series solution to calculate the coupling coefficient of a device, $K(z)$, as a function of the target frequency response in reflection, 
$S_{11}(\beta)$. Particularizing this series solution for the beginning of the device, $z=0$, it can be seen that just the first term of the series is not null, obtaining:

$$
K(z=0)=-2 \cdot F\left(\tau=0^{+}\right)
$$

being $F\left(\tau=0^{+}\right)$the right-hand limit of $F(\tau)$ at $\tau=0$, and

$$
F(\tau)=\frac{1}{2 \pi} \int_{-\infty}^{\infty} S_{11}(\beta) \cdot e^{j \cdot \beta \cdot \tau} \cdot d \beta
$$

i.e., $F(\tau)$ is the inverse Fourier Transform of the frequency response in reflection of the device, $S_{11}(\beta)$.

As a result of causality $F\left(\tau=0^{-}\right)=0$, and $F(\tau)$ will have a step discontinuity at $\tau=0$. Thus, to evaluate the inverse Fourier integral at that discontinuity, the average value at $\tau=$ $0^{-}$and $\tau=0^{+}$needs to be used [75]:

$$
F(\tau=0)=\frac{F\left(\tau=0^{+}\right)+F\left(\tau=0^{-}\right)}{2}=\frac{F\left(\tau=0^{+}\right)}{2}
$$

and taking into account (13) and (14) it can be obtained:

$$
\begin{gathered}
F\left(\tau=0^{+}\right)=2 \cdot F(\tau=0)= \\
=\left.2 \cdot \frac{1}{2 \pi} \int_{-\infty}^{\infty} S_{11}(\beta) \cdot e^{j \cdot \beta \cdot \tau} \cdot d \beta\right|_{\tau=0}= \\
=\frac{1}{\pi} \int_{-\infty}^{\infty} S_{11}(\beta) \cdot d \beta
\end{gathered}
$$

Incorporating this result into (12) it can be finally obtained:

$$
K(z=0)=-\frac{2}{\pi} \int_{-\infty}^{\infty} S_{11}(\beta) \cdot d \beta
$$

It is interesting to note that (16) allows us to calculate the coupling coefficient at the origin of the device, $z=0$, as a function of the reflection coefficient also at the origin of the device.

In order to get the most efficient numerical implementation of (16), the property satisfied by any real physical device $S_{i j}(-f)=S_{i j}^{*}(f)$ will be taken into account. It implies in our case that $S_{11}(-\beta)=S_{11}^{*}(\beta)$. Therefore, expression (16) can be finally rewritten as:

$$
K(z=0)=-\frac{4}{\pi} \int_{0}^{\infty} \mathcal{R} e\left\{S_{11}(\beta)\right\} \cdot d \beta
$$

Now, the Riccati equation will be used to propagate the target reflection coefficient along the device, to allow us to peel off the synthesized layer. The Riccati equation can be obtained from the simplified system of coupled-mode equations employed to model the 1D-EBG device (1). We introduce the reflection coefficient parameter $\rho$, defined as:

$$
\rho(z, \beta)=\frac{a^{-}(z, \beta)}{a^{+}(z, \beta)}
$$

and taking its derivative it results in:

$$
\frac{d \rho}{d z}=\frac{\left(\frac{d a^{-}}{d z} \cdot a^{+}-\frac{d a^{+}}{d z} \cdot a^{-}\right)}{\left(a^{+}\right)^{2}}
$$

Introducing the simplified system of couple-mode equations (1) we obtain:

$$
\frac{d \rho}{d z}=\frac{\left(j \cdot \beta \cdot a^{-}+K \cdot a^{+}\right) \cdot a^{+}-\left(-j \cdot \beta \cdot a^{+}+K \cdot a^{-}\right) \cdot a^{-}}{\left(a^{+}\right)^{2}}
$$

and reorganizing the equation it can be rewritten as:

$$
\frac{d \rho}{d z}=j \cdot \beta \cdot \frac{a^{-}}{a^{+}}+K+j \cdot \beta \cdot \frac{a^{-}}{a^{+}}-K \cdot \frac{\left(a^{-}\right)^{2}}{\left(a^{+}\right)^{2}}
$$

Recalling that the reflection coefficient $\rho$ is defined as (18), the expression can be finally rewritten as the so-called Riccati equation, [76]:

$$
\frac{d \rho}{d z}=2 \cdot j \cdot \beta \cdot \rho+K \cdot\left(1-\rho^{2}\right)
$$

Once the coupling coefficient at the origin of the device, $K(0)$, is calculated using (17), the Riccati equation (20) will be employed to propagate the target reflection coefficient spectrum along the first infinitesimal layer of the device. With this new target spectrum, the first infinitesimal layer is peeled off and the origin of the rest of the device is shifted to the next layer. Then, the next point of the coupling coefficient can be calculated using (17). Following in an iterative manner, i.e., propagating the target spectrum with (20) and calculating the next value of the coupling coefficient by means of (17), the entire coupling coefficient, $K(z)$, for the $1 \mathrm{D}-\mathrm{EBG}$ device is synthesized.

The equations that relate the physical dimensions of the device and the desired coupling coefficient will depend on the technology used [63]. For our case of planar microwave technology with TEM or quasi-TEM operation mode, the characteristic impedance $Z_{0}(z)$ can be used as an intermediate parameter [61], [62]:

$$
K(z)=\frac{-1}{2 \cdot Z_{0}(z)} \cdot \frac{d Z_{0}(z)}{d z}
$$

This equation can be solved analytically. Thus, knowing the coupling coefficient required between the forward and backward travelling waves of the fundamental TEM or quasiTEM mode, an analytical expression for the characteristic impedance required for the device can be deduced as:

$$
Z_{0}(z)=Z_{0}(0) \cdot e^{-2 \cdot \int_{0}^{z} K(g) \cdot d g}
$$


being $Z_{0}(0)$ the value of the characteristic impedance at the input port, and $g$ just a dummy variable to calculate the integral.

\section{Schematic Design Procedure}

Summing up, the synthesis methodology proposed in this paper for spurious-free 1D-EBG structures with fully controlled parameters consists of seven steps:

a) The period of the perturbation, $\Lambda$, is fixed from the central frequency of the stopband, $f_{0}$ (frequency with maximum rejection level). This is done using (4).

b) The order $N$ is estimated with (9) (valid for Chebyshev responses) from the required frequency specifications. Inspecting (11), it can be seen that if the input and output ports are specified as equal, only odd values of $N$ should be selected. In this situation use the upper-closest odd value given by (9).

c) Using the calculated order $N$, the required frequency specifications and (10), the list of characteristic impedances $Z_{i}$ for the classical Chebyshev periodic filter is obtained. The values calculated for the $Z_{i}$ using the analytical expressions are just approximate. If higher precision is required for the $Z_{i}$ values, the accurate numerical solution provided by Keysight ${ }^{\mathrm{TM}}$ Genesys S/Filter software tool will be employed.

d) The frequency response of the classical periodic filter is obtained by multiplying the transfer matrices of the $N$ cascaded transmission line sections.

e) The previously calculated frequency response is modified by nulling the values of the $S_{11}(f)$ above $2 \cdot f_{0}$, in order to obtain a target $1 \mathrm{D}-\mathrm{EBG}$ response with a single spurious-free stopband.

f) The continuous layer peeling algorithm of (17) and (20) is applied to synthesize the required coupling coefficient, $K(z)$, to obtain the desired 1D-EBG structure that satisfies the target frequency response fulfilling all the specifications.

g) The characteristic impedance profile of the designed 1DEBG structure, as a function of the propagation direction, $Z_{0}(z)$, is calculated from the required coupling coefficient, $K(z)$, by means of (22).

\section{Design EXAMPLE: PROOF OF CONCEPT}

The feasibility of the synthesis methodology explained in the previous section will be tested now with the design of a 1D-EBG structure in microstrip technology, free of spurious stopbands, and with all the performance parameters under control. The design requirements are: central frequency of the stopband $f_{0}=1 \mathrm{GHz}$, maximum attenuation of $30 \mathrm{~dB}$, fractional bandwidth of the stopband larger than $100 \%(B W=$ $1.11 \mathrm{GHz}$ ), and return loss level out of the forbidden band of $20 \mathrm{~dB}$.

In first place, a design using just the analytical method explained in Section II.A will be done. The substrate employed has thickness $h=1.524 \mathrm{~mm}$, dielectric constant $\varepsilon_{r}=3.5$, and $50 \Omega$-input and output ports. Using (4), (5) and (6) the characteristic parameters for the sinusoidal coupling coefficient solution employed are calculated: $\Lambda=9.05 \mathrm{~cm}$, $A=30.56 \mathrm{~m}^{-1}, \quad L=27.15 \mathrm{~cm}$. The resulting coupling

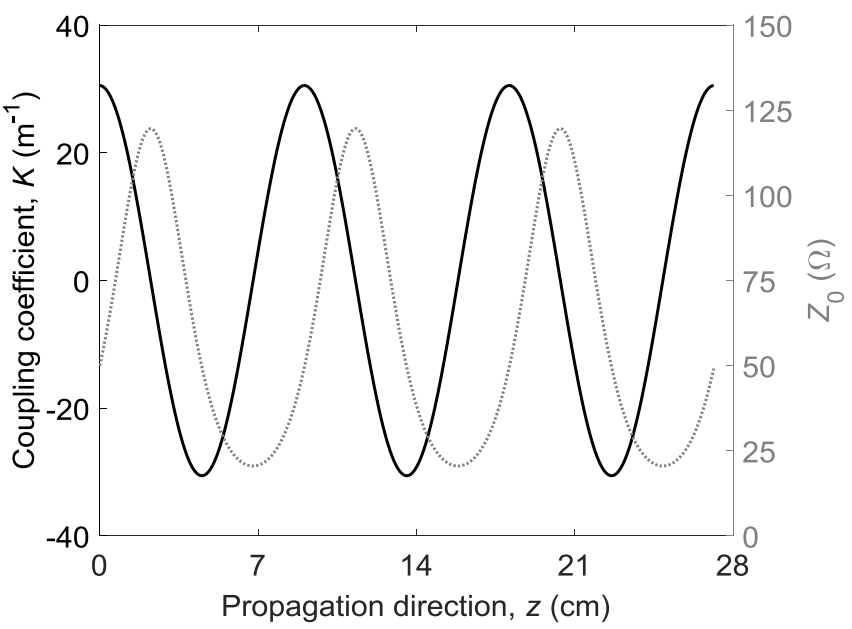

Fig. 4. Coupling coefficient (black line) and characteristic impedance (grey dotted line) along the propagation direction of the 1D-EBG structure designed using the analytical method of Section II.A.

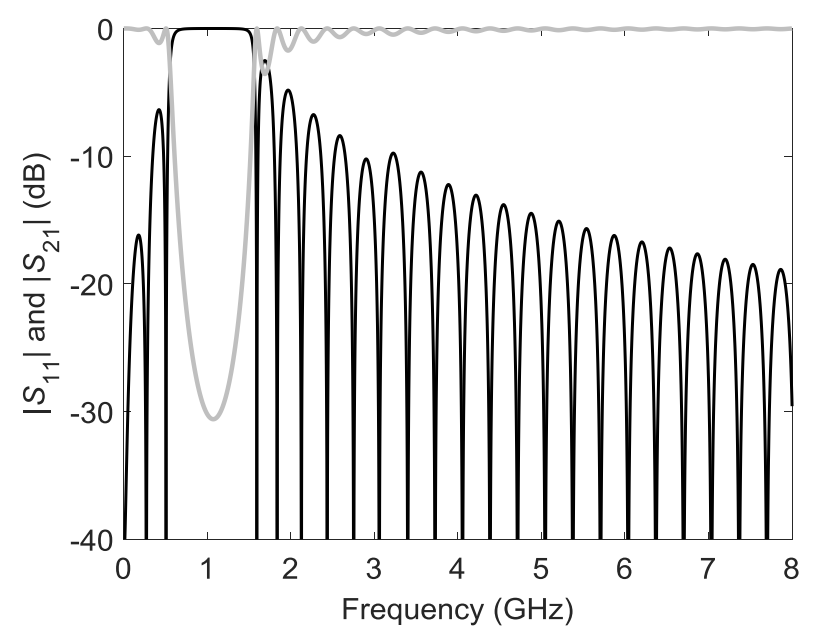

Fig. 5. Magnitude of the $S_{11}$-parameter (black line) and $S_{21}$-parameter (grey line) of the 1D-EBG structure designed using the analytical method of Section II.A.

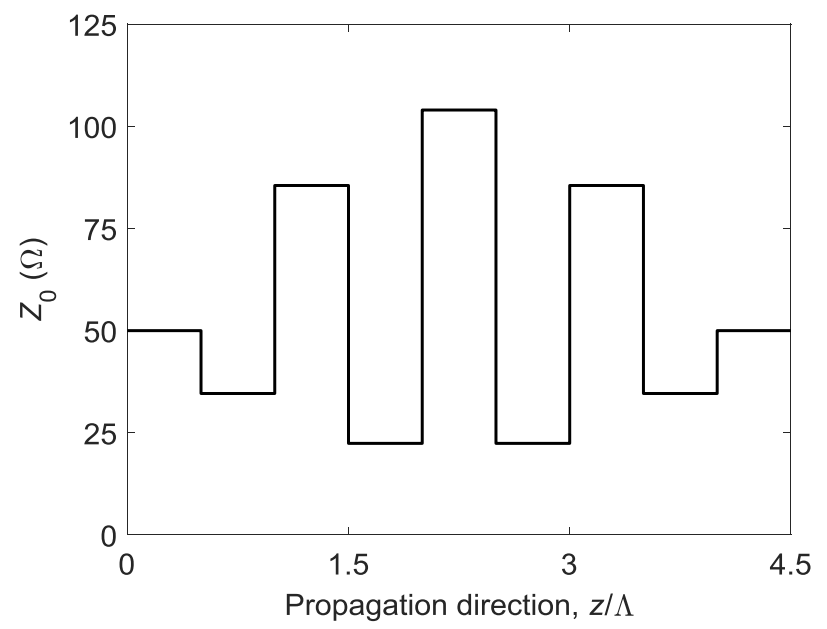

Fig. 6. Characteristic impedance of the 7th-section classical Chebyshev periodic filter along the propagation direction. 


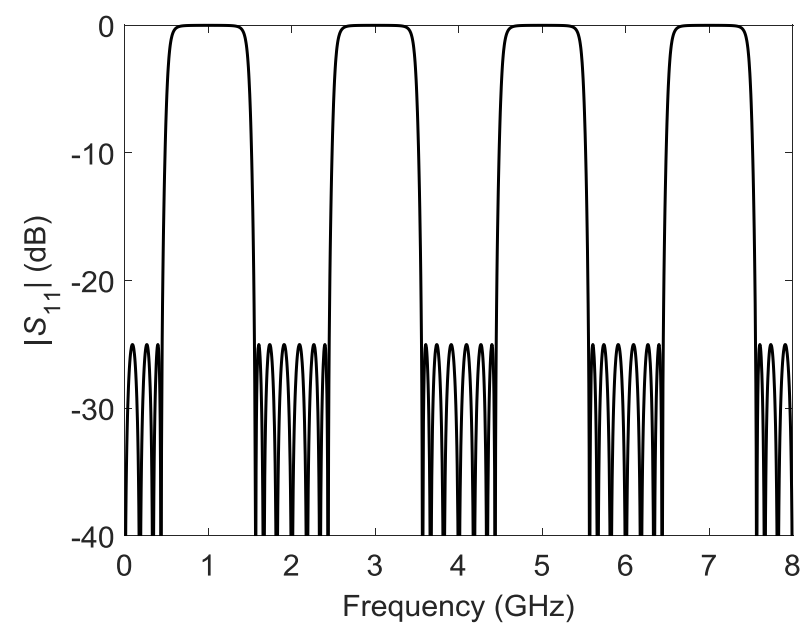

(a)

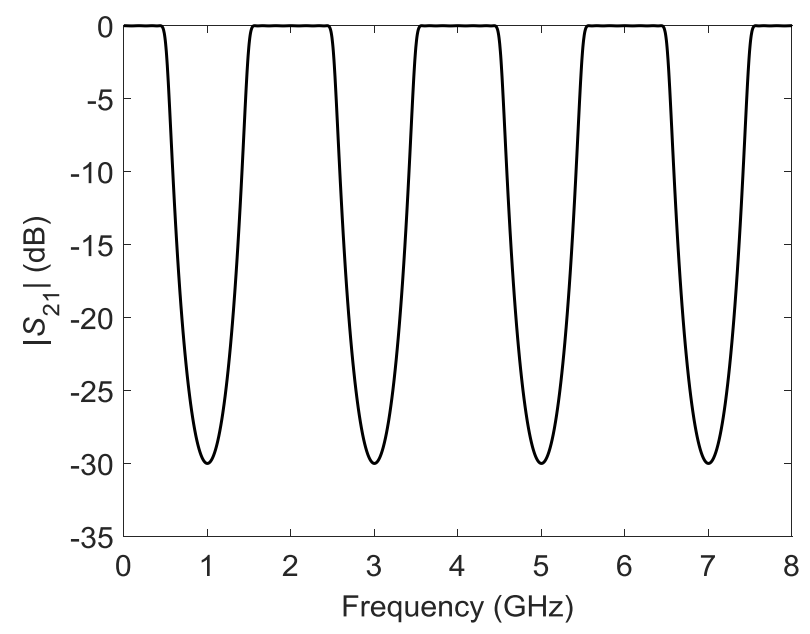

(b)

Fig. 7. Magnitude of the frequency response of the 7th-section classical Chebyshev periodic filter: the $S_{11}$ (a) and $S_{21}$ (b) parameters are given.

coefficient and characteristic impedance profile (obtained with (22)) for the 1D-EBG microstrip structure are depicted in Fig. 4. The EBG structure has been simulated using the coupledmode equations (1) and the results are given in Fig. 5. As it can be seen, the design requirements are approximately satisfied except for the return loss level out of the forbidden band, which has a worst-case value of around $2.5 \mathrm{~dB}$. Unfortunately, that value is far away from the required $20 \mathrm{~dB}$, and thus the $1 \mathrm{D}-\mathrm{EBG}$ obtained is not valid.

Having demonstrated that the analytical method explained in Section II.A is not able to satisfy the required return loss values, the novel synthesis methodology proposed in this paper will be employed now. Following the procedure explained in Section II, the first step is to design the Nth-section classical periodic filter. The length of each transmission line section will be $\Lambda / 2$, where $\Lambda$ is calculated with (4). Selecting the Chebyshev response, assigning a security margin to the return loss level $R L=25 \mathrm{~dB}$, and using (9) the required filter order $N=7$ is obtained. Using (10) and imposing a value of $Z_{0}=50 \Omega$ for the

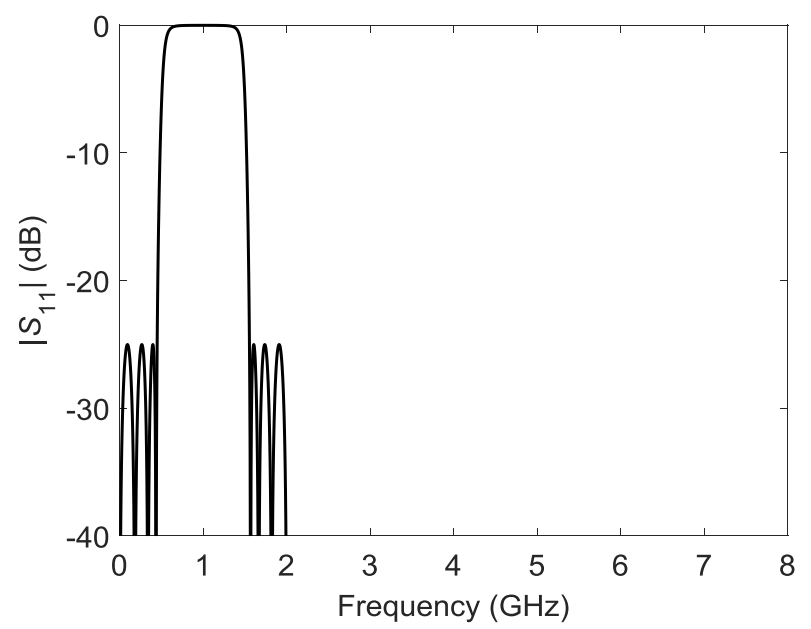

(a)

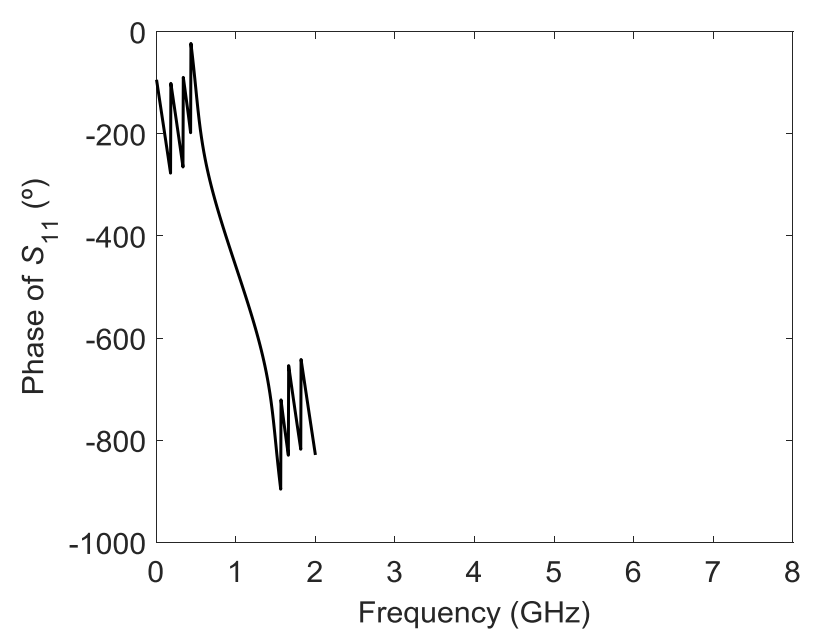

(b)

Fig. 8. Target magnitude (a) and phase (b) of the $S_{11}$-parameter for the 1DEBG structure designed as an example.

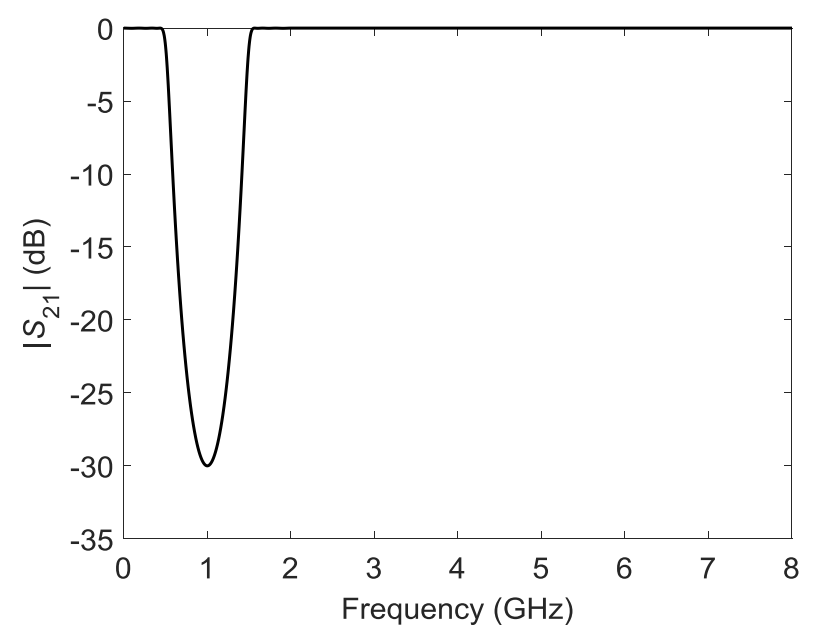

Fig. 9. Target $\left|S_{21}\right|$-parameter for the 1D-EBG structure designed as an example. 
input port, the characteristic impedances for each section are: $Z_{1}=Z_{7}=34.48 \Omega, Z_{2}=Z_{6}=80.47 \Omega, Z_{3}=Z_{5}=21.43 \Omega$, $Z_{4}=108.49 \Omega$. More accurate values are obtained if the Keysight ${ }^{\mathrm{TM}}$ Genesys S/Filter software tool is employed to perform the numerical calculations: $Z_{1}=Z_{7}=34.61 \Omega, Z_{2}=$ $Z_{6}=85.54 \Omega, \quad Z_{3}=Z_{5}=22.40 \Omega, \quad Z_{4}=104.02 \Omega$. The characteristic impedances are depicted in Fig. 6.

The frequency response of the 7 th-section classical periodic filter is computed by transfer matrix multiplication of ideal transmission line sections of characteristic impedance $Z_{i}$ and length $\Lambda / 2$. The results are shown in Fig. 7, where all the target specifications are satisfied apart from the appearance of the inherent spurious stopbands. The next step is to remove those spurious stopbands by introducing null values in the $S_{11}(f)$ response for frequencies higher than $2 \mathrm{GHz}$. The resulting target frequency response is represented in Fig. 8. and Fig. 9.

From the target frequency response, the coupling coefficient required for the $1 \mathrm{D}-\mathrm{EBG}$ structure can be obtained. To do it, the novel continuous layer peeling synthesis method proposed in

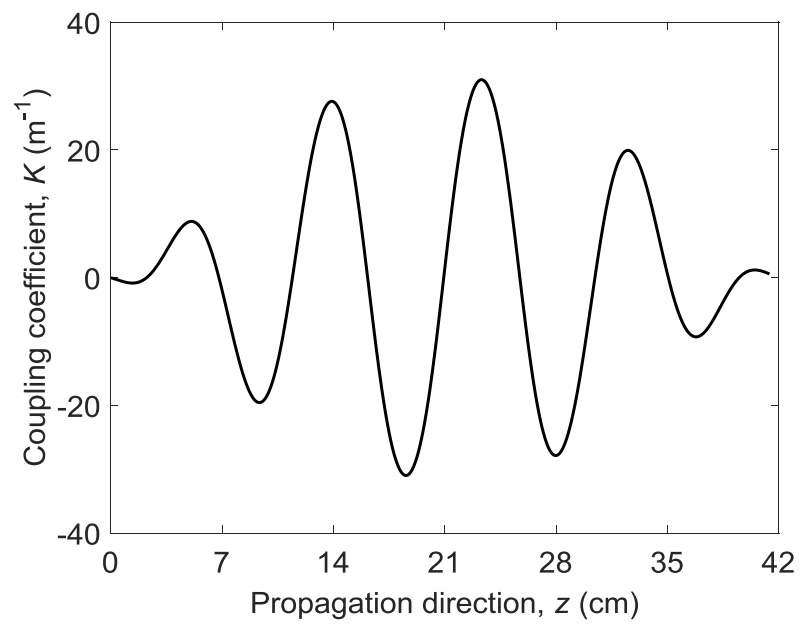

Fig. 10. Coupling coefficient of the 1D-EBG structure designed as an example along the propagation direction.

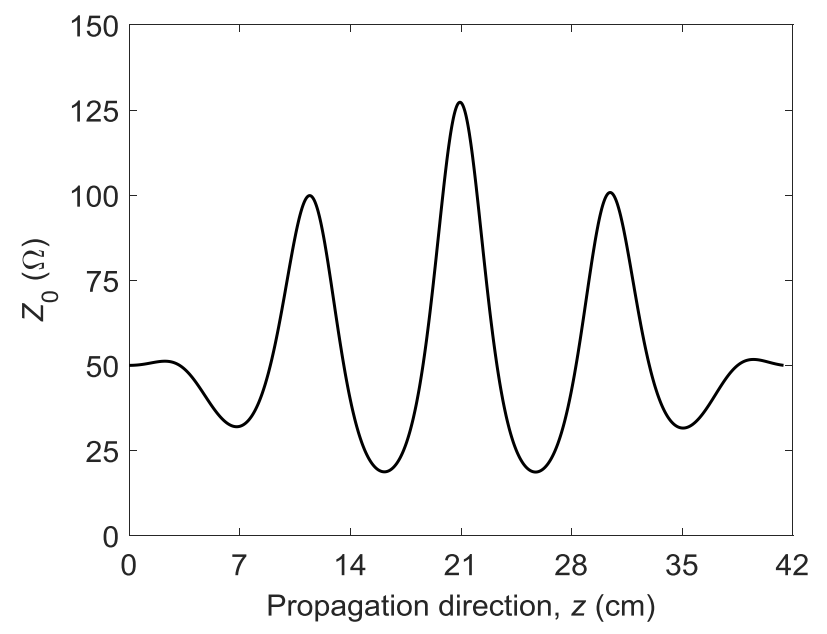

Fig. 11. Characteristic impedance of the 1D-EBG structure designed as an example along the propagation direction.
Subsection II.C will be employed. Thus, from the complex (magnitude and phase) target $S_{11}(f)$, defined numerically from $f=0 \mathrm{GHz}$ to $f=200 \mathrm{GHz}$ every $4 \mathrm{MHz}$, and partially shown in Fig. 8, the $K(0)$ is obtained using (17). With this value, the Ricatti equation (20) is solved using the Matlab ${ }^{\mathrm{TM}}$ ODE solver "ode45.m" function, to peel off the layer propagating $S_{11}(f)$ to obtain the new target reflection coefficient. By means of (17) the next sample of the coupling coefficient is straightforwardly calculated. In a layer by layer iterative routine, the whole coupling coefficient is recovered and shown in Fig. 10. The required characteristic impedance for the microstrip line is finally calculated using (22) and it is displayed in Fig. 11. The physical dimensions of the microstrip 1D-EBG structure are obtained by means of the Keysight ${ }^{\mathrm{TM}}$ Linecalc software tool. A slight redistribution is applied to the $z$-axis (propagation direction) in order to compensate for the variation of the effective dielectric constant along the microstrip structure. The details of the simple procedure employed can be read in [69]. The metallic-strip width along the propagation direction of the final structure is depicted in Fig. 12 (a Rogers RO3035 ${ }^{\mathrm{TM}}$ substrate has been used with thickness $h=1.524 \mathrm{~mm}$, dielectric constant $\varepsilon_{r}=3.5$, and $50 \Omega$-input and output ports). The final length of the device is $2.28 \cdot \lambda$ (with $\lambda$ the guided wavelength at the maximum rejection frequency, $f_{0}$ ). A photograph of the fabricated prototype is shown in Fig. 13. The designed 1D-EBG structure has been simulated using Keysight $^{\mathrm{TM}}$ ADS Momentum and measured with an Agilent ${ }^{\mathrm{TM}}$ 8722 Vector Network Analyzer. The simulated and measured results are depicted in Fig. 14 and Fig. 15. A detail of the measured frequency response around the rejected band is given in Fig. 16 and Fig. 17, together with the target response.

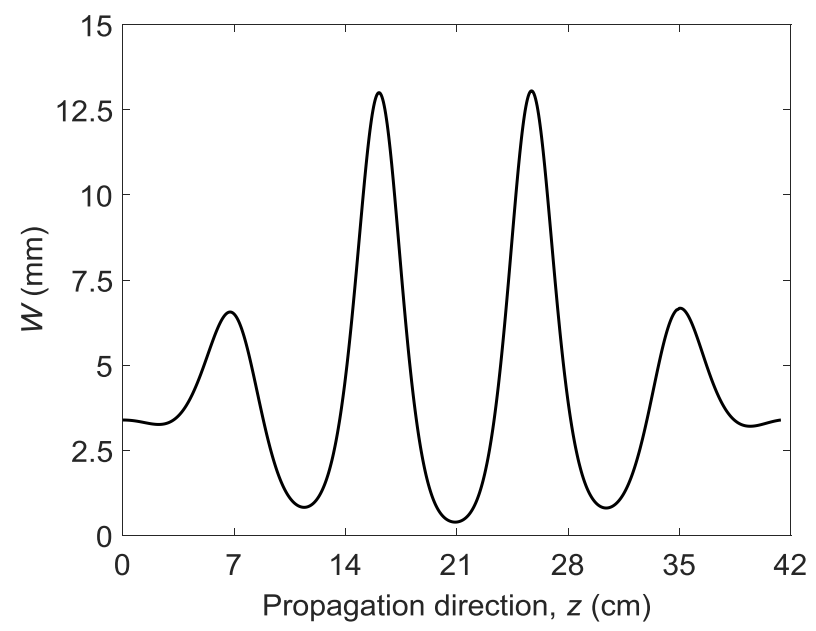

Fig. 12. Physical dimensions of the 1D-EBG structure designed as an example: microstrip line width along the propagation direction.

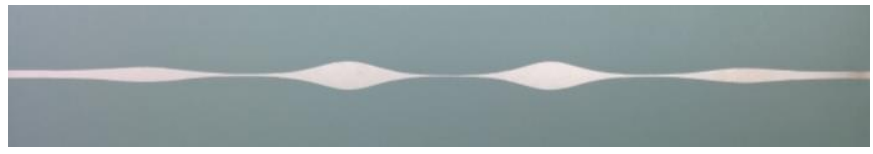

Fig. 13. Photograph of the fabricated 1D-EBG structure designed as an example. 


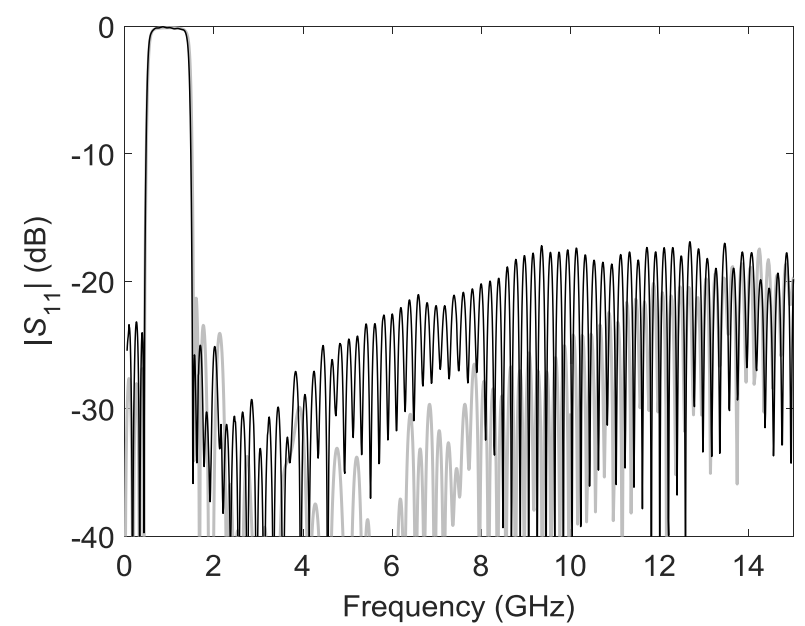

Fig. 14. Simulated (grey line) and measured (black line) magnitude of the $S_{11}$-parameter of the $1 \mathrm{D}$-EBG structure designed as an example.

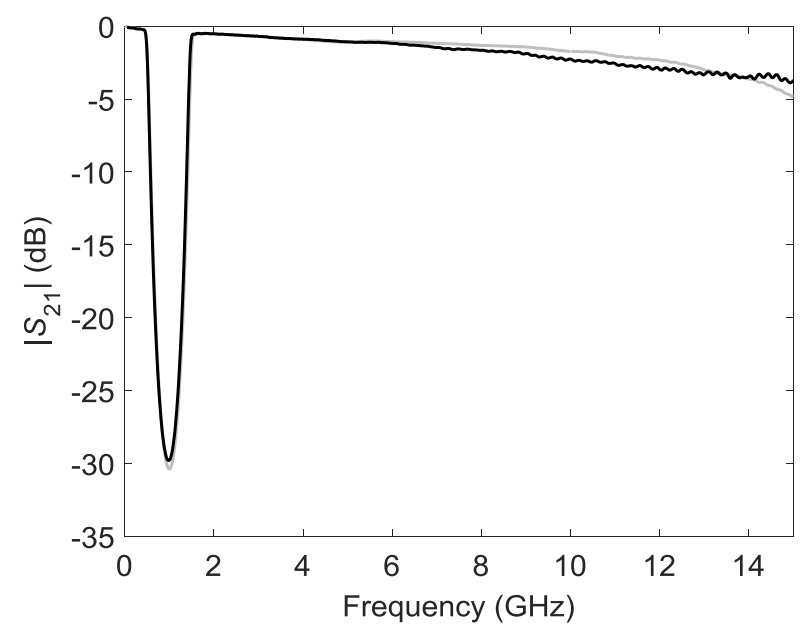

Fig. 15. Simulated (grey line) and measured (black line) magnitude of the $S_{21}$-parameter of the 1D-EBG structure designed as an example.

The excellent agreement found between the target response, the simulations, and the measurements confirms the accuracy of the novel synthesis methodology proposed. Specifically, no spurious stopbands appear up to the 15 th harmonic $\left(15 \cdot f_{0}\right)$, and the return loss level out of the stopband is better than $20 \mathrm{~dB}$ in measurement up to $8 \mathrm{GHz}\left(8 \cdot f_{0}\right)$. The return loss level decreases beyond that frequency due to the connectors and to the dispersion of the microstrip line, but it remains better than $17 \mathrm{~dB}$ in measurement up to $15 \mathrm{GHz}\left(15 \cdot f_{0}\right)$. Focusing on Fig. 16 and Fig. 17 it can be seen that the target specifications are achieved in measurement with remarkable accuracy: central frequency of the stopband, $f_{0}=1 \mathrm{GHz}$, bandwidth of the stopband, $B W=1.1 \mathrm{GHz}$, maximum rejection of $30 \mathrm{~dB}$, and return loss level out of the forbidden band better $20 \mathrm{~dB}$. The small discrepancies found can be attributed to the fabrication tolerances, to the uncertainty in the dielectric constant of the substrate and to the connectors.

To finish this section, the results obtained in our example will be compared with the state of the art in filters based on EBG structures. A very interesting work has been recently published [77] where a 1D-EBG filter with rejection level better than

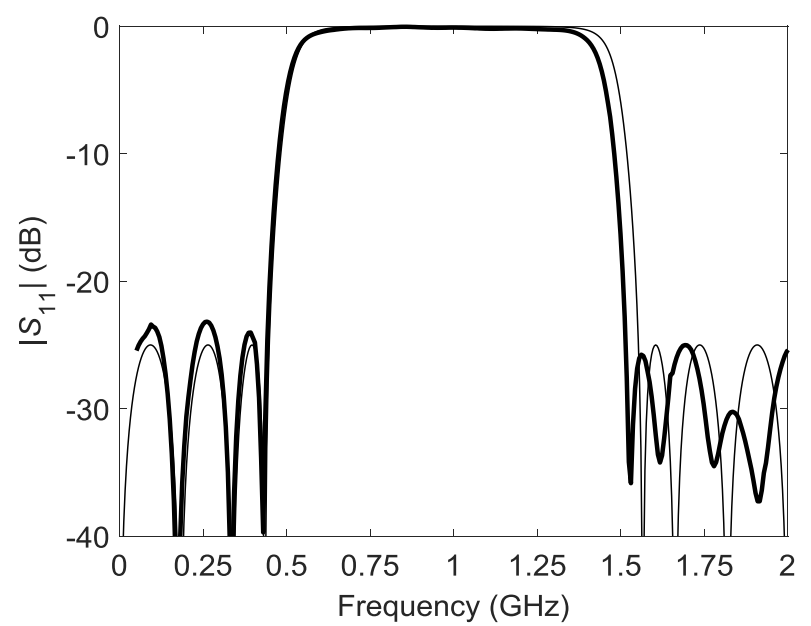

(a)

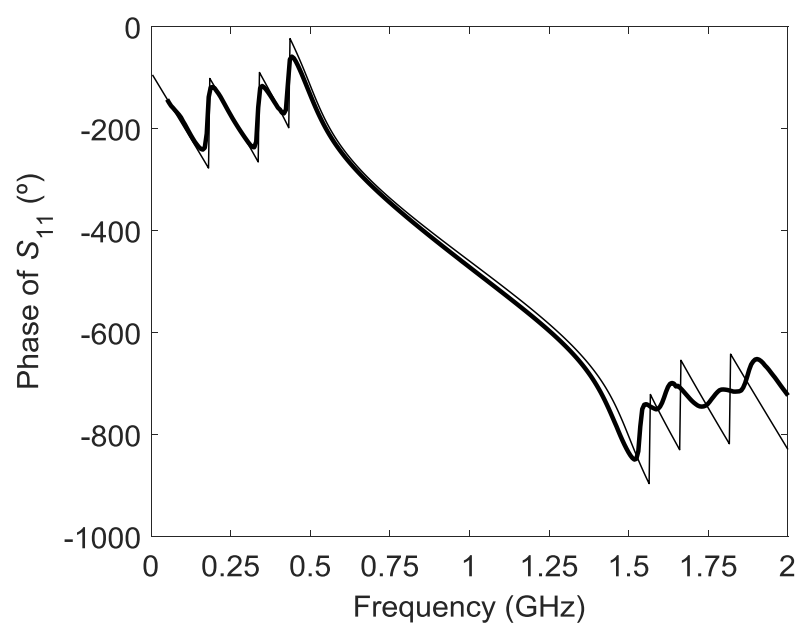

(b)

Fig. 16. Target (thin black line) and measured (thick black line) magnitude (a) and phase (b) of the $S_{11}$-parameter of the 1D-EBG structure designed as an example.

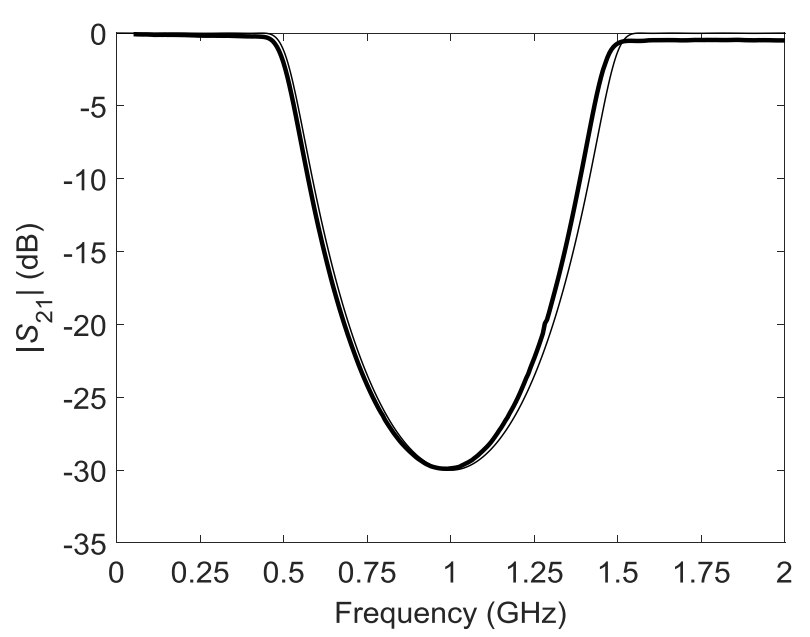

Fig. 17. Target (thin black line) and measured (thick black line) magnitude of the $S_{21}$-parameter of the 1D-EBG structure designed as an example. 
$80 \mathrm{~dB}$ was proposed, although it lacks in return loss level in the passband (only $10 \mathrm{~dB}$, not enough for many applications) and straightness in the design procedure. A comparison of $12 \mathrm{EBG}$ devices covering many authors, years, and types of EBG structures is also included in that paper. It is interesting to note that the best return loss level achieved in the comparative table [[77], Table 2] is $15.5 \mathrm{~dB}$, and the structure presents only $25 \mathrm{~dB}$ of rejection [78]. The next paper with best return loss is [79] with $13 \mathrm{~dB}$, albeit it provides a very competitive EBG structure in terms of size. Another paper with a performance-review of 1D-EBG filters is [79], where 8 papers are compared [[79], Table II]. The best return loss level in that comparison is $15 \mathrm{~dB}$ corresponding to [54], with a device of $40 \mathrm{~dB}$ rejection level but with fractional bandwidth of just $50 \%$, clearly insufficient for many applications.

Finally, it must be highlighted that telecommunication and radar systems, as well as material characterization setups, can be profited by the accurate and powerful design methodology proposed in this paper. Actually, in our proof of concept design example, a 1D-EBG structure has been implemented with rejection bandwidth larger than $100 \%$, maximum rejection of $30 \mathrm{~dB}$, no spurious stopbands up to the 15 th harmonic $\left(15 \cdot f_{0}\right)$ in measurement, and with return loss level better than $20 \mathrm{~dB}$ in measurement for all the frequencies out of the stopband up to the 8 th harmonic $\left(8 \cdot f_{0}\right)$.

\section{CONCLUSION}

A novel synthesis methodology for the design of 1D-EBG structures with no spurious stopbands and with full control over all the design parameters, has been proposed and successfully tested. The idea rests on the use of the coupled-mode theory to model the electromagnetic behavior of the 1D-EBG structures, the classical filter theory of periodic structures to obtain an optimum target frequency response, and an inverse scattering synthesis method to finally synthesize the EBG structure. A straightforward design procedure has been described, with clear steps, and a detailed example of application has been given.

The remarkable effort done by many authors to minimize the ripple (return loss level) in the passband of EBG structures, has been discussed in the introduction of the paper with a careful review of the literature. Additionally, the best results presented in the literature for 1D-EBG structures have been compared with the return loss level obtained in our design example, highlighting the importance of the $20 \mathrm{~dB}$ value set as target and achieved in measurement in our prototype up to the 8th harmonic.

Filters, amplifiers, oscillators, mixers, power dividers, couplers and sensors are advanced components involved in cutting-edge telecommunication, radar or sensing systems, which can be benefited by the novel EBG structures proposed in this paper.

\section{REFERENCES}

[1] R. B. Hwang, Periodic Structures: Mode-Matching Approach and Applications in Electromagnetic Engineering, John Wiley \& Sons Singapore Pte. Ltd, 2013.
[2] J. D. Joannopoulos, S. G. Johnson, J. N. Winn, and R. D. Meade, Photonic Crystals: Molding the flow of light, Princeton, NJ: $2^{\text {nd }}$ Ed. Princeton University Press, 2008.

[3] F. Martín, Artificial Transmission Lines for RF and Microwave Applications. Hoboken, NJ, USA: Wiley, 2015.

[4] E. Yablonovitch, "Inhibited spontaneous emission in solid-state physics and electronics," Phys. Rev. Lett., vol. 58, no. 20, pp. 2059-2062, May 1987.

[5] S. John, "Strong localization of photons in certain disordered dielectric superlattices," Phys. Rev. Lett., vol. 58, no. 23, pp. 2486-2489, June 1987.

[6] N. Engheta, and R.W. Ziolkowski, Metamaterials: Physics and Engineering Explorations, Wiley-IEEE Press 2006.

[7] D. Sievenpiper, Lijun Zhang, R. F. J. Broas, N. G. Alexopolous, and E. Yablonovitch, "High-impedance electromagnetic surfaces with a forbidden frequency band," IEEE Trans. Microw. Theory Techn., vol. 47, no. 11, pp. 2059-2074, Nov. 1999.

[8] R. Gonzalo, P. De Maagt, and M. Sorolla, "Enhanced patch-antenna performance by suppressing surface waves using photonic-bandgap substrates," IEEE Trans. Microw. Theory Techn., vol. 47, no. 11, pp. 2131-2138, Nov 1999.

[9] B. P. Smyth, S. Barth, and A. K. Iyer, "Dual-Band Microstrip Patch Antenna Using Integrated Uniplanar Metamaterial-Based EBGs," IEEE Trans. Antennas Propag., vol. 64, no. 12, pp. 5046-5053, Dec. 2016.

[10] D. Nesic, and A. Nesic, "Band-stop microstrip PBG filter with sinusoidal variation of the characteristic impedance and without etching in the group plane," Microw. Opt. Technol. Lett. 29, pp. 418-420, June 2001.

[11] F. Falcone, T. Lopetegi, and M. Sorolla, "1-D and 2-D Photonic Band Gap Microstrip Structures," Microw. Opt. Technol. Lett. 22, pp. 411-412, Aug. 1999.

[12] Y. Q. Fu, G. H. Zhang, and N. C. Yuan, "A novel PBG coplanar waveguide," IEEE Microw. Compon. Lett., vol. 11, no. 11, pp. 447-449, Nov. 2001.

[13] C. C. Cutler, "Corrugated wave guide devices," U.S. Patent 2912695, Nov. 10, 1959.

[14] I. Arnedo et al., "Ku-band high-power lowpass filter with spurious rejection," Electron. Lett. 42, pp. 1460-1461, Dec. 2006.

[15] N. F. Kovalev, I. M. Orlova, and M. I. Petelin, "Wave Transformation in a multi-mode waveguide with corrugated walls," Radio Phys. Quantum Electron., vol. 11, pp. 449-450, May 1968.

[16] A. A. Oliner, and W. Rotman, "Periodic Structures in Trough Waveguides," IEEE Trans. Microw. Theory Techn., vol. 7, no. 1, pp. 134142, Jan. 1959.

[17] O. R. Asfar, and A. H. Nayfeh, "Circular waveguide with sinusoidally perturbed wall," IEEE Trans. Microw. Theory Techn., vol.23, no. 9, pp. 728- 734, Sept. 1975.

[18] E. D. Caballero et al., "Mapping smooth profile H-plane rectangular waveguide structures to substrate integrated waveguide technology," Electron. Lett., vol. 50, no. 15, pp. 1072-1074, Jul. 2014.

[19] J. de Dios Ruiz, F. L. Martínez-Viviente, A. Alvarez-Melcon, and J. Hinojosa, "Substrate Integrated Waveguide (SIW) With Koch Fractal Electromagnetic Bandgap Structures (KFEBG) for Bandpass Filter Design," IEEE Microw. Compon. Lett., vol. 25, no. 3, pp. 160-162, Mar. 2015.

[20] L. S. Wu et al., "A New Type of Periodically Loaded Half-Mode Substrate Integrated Waveguide and Its Applications," IEEE Trans. Microw. Theory Techn., vol. 58, no. 4, pp. 882-893, Apr. 2010.

[21] T. Decoopman, X. Melique, O. Vanbesien, and D. Lippens, "A taper filtering finline at millimeter wavelengths for broadband harmonic multiplication," IEEE Microw. Compon. Lett., vol. 13, no. 5, pp. 172-174, May 2003.

[22] Y. Qian, V. Radisic, and T. Itoh, "Simulation and experiment of photonic band-gap structures for microstrip circuits," in Proc. 1997 Asia-Pacific Microwave Conf., 1997, pp. 585-588 vol. 2.

[23] V. Radisic, Y. Qian, and T. Itoh, "Novel architectures for high-efficiency amplifiers for wireless applications," IEEE Trans. Microw. Theory Techn., vol. 46, no. 11, pp. 1901-1909, Nov. 1998.

[24] J. S. Lim, H. S. Kim, J. S. Park, D. Ahn, and S. Nam, "A power amplifier with efficiency improved using defected ground structure," IEEE Microw. Compon. Lett., vol. 11, no. 4, pp. 170-172, Apr. 2001.

[25] Q. Xue, K. M. Shum, and C. H. Chan, "Novel Oscillator Incorporating a Compact Microstrip Resonant Cell", IEEE Microw. Compon. Lett., vol. 11, no. 5, pp. 202-204, May 2001.

[26] Y. T. Lee, J. S. Lim, J. S. Park, D. Ahn, and S. Nam, "A novel phase noise reduction technique in oscillators using defected ground structure," IEEE Microw. Compon. Lett., vol. 12, no. 2, pp. 39-41, Feb. 2002. 
[27] F. R. Yang, Y. Qian, and T. Itoh, "A novel uniplanar compact PBG structure for filter and mixer applications," in 1999 IEEE MTT-S International Microwave Symposium Digest (Cat. No.99CH36282), Anaheim, CA, USA, 1999, pp. 919-922 vol.3.

[28] M. Zhou, J. Xu, S. D. Luo, and L. J. Xue, "Ka-band fourth harmonic mixer with 1-D EBG structure," Hongwai Yu Haomibo Xuebao/Journal of Infrared and Millimeter Waves, 25 (2), pp. 147-149, Apr. 2006.

[29] M. Chudzik et al., "Microstrip coupled-line directional coupler with enhanced coupling based on EBG concept," Electron. Lett., vol. 47, no. 23, pp. 1284-1286, Nov. 2011.

[30] M. Chudzik et al., "Design of EBG microstrip directional coupler with high directivity and coupling," 2012 42nd European Microwave Conf., Amsterdam, 2012, pp. 483-486.

[31] B. L. Ooi, "Compact EBG in-phase hybrid-ring equal power divider," IEEE Trans. Microw. Theory Techn., vol. 53, no. 7, pp. 2329-2334, July 2005.

[32] B. Garcia-Banos, F. Cuesta-Soto, A. Griol, J. M. Catala-Civera, and J. Pitarch, "Enhancement of Sensitivity of Microwave Planar Sensors With EBG Structures," IEEE Sensors Journal, vol. 6, no. 6, pp. 1518-1522, Dec. 2006.

[33] S. Subbaraj, V. S. Ramalingam, M. Kanagasabai, E. F. Sundarsingh, Y. P. Selvam, and S. Kingsley, "Electromagnetic Nondestructive Material Characterization of Dielectrics Using EBG Based Planar Transmission Line Sensor," IEEE Sensors Journal, vol. 16, no. 19, pp. 7081-7087, Oct.1, 2016.

[34] A. Cismaru, M. Aldrigo, A, Radoi, and M. Dragoman, "Carbon nanotubebased electromagnetic band gap resonator for $\mathrm{CH} 4$ gas detection," Journal of Applied Physics, 119 (12), art. no. 124504, 2016.

[35] D. Ahn, J. S. Park, C. S. Kim, J. Kim, Y. Qian, and T. Itoh, "A design of the low-pass filter using the novel microstrip defected ground structure," IEEE Trans. Microw. Theory Techn., vol. 49, no. 1, pp. 86-93, Jan 2001.

[36] N. C. Karmakar, and M. N. Mollah, "Investigations into nonuniform photonic-bandgap microstrip line low-pass filters," IEEE Trans. Microw. Theory Techn., vol. 51, no. 2, pp. 564-572, Feb. 2003.

[37] F. R. Yang, Y. Qian, and T. Itoh, "A novel uniplanar compact PBG structure for filter and mixer applications," 1999 IEEE MTT-S International Microwave Symp., Anaheim, CA, pp. 919-922, June 1999.

[38] T. Kim, and C. Seo, "A novel photonic bandgap structure for low-pass filter of wide stopband," IEEE Microw. Guided Wave Lett., vol. 10, no. 1, pp. 13-15, Jan. 2000.

[39] T. Lopetegi et al., "New microstrip "Wiggly-Line" filters with spurious passband suppression," IEEE Trans. Microw. Theory Techn., vol. 49, no. 9, pp. 1593-1598, Sep 2001.

[40] A. Alphones, and J. Cheng, "Compact interdigitated microstrip bandpass filter with meandered EBGs," 2009 European Microwave Conf. (EuMC), Rome, 2009, pp. 439-443.

[41] Yu Ji, X. S. Yao, and L. Maleki, "High-Q whispering gallery mode dielectric resonator bandpass filter with microstrip line coupling and photonic bandgap mode-suppression," IEEE Microw. Guided Wave Lett., vol. 10, no. 8, pp. 310-312, Aug. 2000.

[42] F. R. Yang, K. P. Ma, Y. Qian, and T. Itoh, "A Uniplanar Compact Photonic-Bandgap (UC-PBG) Structure and Its Applications for Microwave Circuits," IEEE Trans. Microw. Theory Techn., vol. 47, no. 8, pp. 1509-1514, Aug. 1999.

[43] F. Martín, J. Bonache, I. Gil, F. Falcone, T. Lopetegi, M.A.G. Laso and M. Sorolla, "Compact spurious free CPW band pass filters based on electromagnetic bandgap structures," Microw. Opt. Technol. Lett., vol. 40, no. 2, pp. 146-148, Jan. 2004.

[44] J. García-García, J. Bonache, and F. Martín, "Application of Electromagnetic Bandgaps to the design of Ultra-Wide Bandpass Filters with good out-of-band performance," IEEE Trans. Microw. Theory Tech., vol. 54, no. 12, pp. 4136-4140, Dec. 2006.

[45] M. Orellana, J. Selga, P. Vélez, M. Sans, A. Rodríguez, J. Bonache, V. Boria and F. Martín, "Design of capacitively-loaded coupled line bandpass filters with compact size and spurious suppression", IEEE Trans. Microw. Theory Tech., vol. 65, no. 4, pp. 1235-1248, April 2017.

[46] V. Radisic, Y. Qian, R. Coccioli, and T. Itoh, "Novel 2-D photonic bandgap structure for microstrip lines," IEEE Microw. Guided Wave Lett., vol. 8, no. 2, pp. 69-71, Feb 1998.

[47] I. Rumsey, M. Piket-May, and P. K. Kelly, "Photonic bandgap structures used as filters in microstrip circuits," IEEE Microw. Guided Wave Lett., vol. 8, no. 10, pp. 336-338, Oct 1998.

[48] L. Kurra, M. P. Abegaonkar, A. Basu, and S. K. Koul, "Switchable and Tunable Notch in Ultra-Wideband Filter Using Electromagnetic Bandgap
Structure," IEEE Microw. Compon. Lett., vol. 24, no. 12, pp. 839-841, Dec. 2014.

[49] F. Martin et al., "The Beauty of Symmetry: Common-Mode Rejection Filters for High-Speed Interconnects and Band Microwave Circuits," IEEE Microw. Mag., vol. 18, no. 1, pp. 42-55, Jan.-Feb. 2017.

[50] P. Velez, J. Bonache, and F. Martin, "Differential Microstrip Lines with Common-Mode Suppression Based on Electromagnetic Band-Gaps (EBGs)," IEEE Antennas Wireless Propag. Lett., vol. 14, pp. 40-43, Sept. 2015.

[51] J. H. Choi, P. W. C. Hon, and T. Itoh, "Dispersion Analysis and Design of Planar Electromagnetic Bandgap Ground Plane for Broadband Common-Mode Suppression," IEEE Microw. Compon. Lett., vol. 24, no. 11, pp. 772-774, Nov. 2014.

[52] T. Lopetegi, "Photonic bandgap structures in microstrip technology: Study using the coupled mode formalism and applications," Ph.D. dissertation, Public University of Navarre (UPNA), Pamplona-Iruña, Spain, 2002.

[53] S. M. S. Hassan, and M. N. Mollah, "Identical performance from distinct conventional electromagnetic bandgap structures," IET Microwaves Antennas Propag., vol. 10, no. 12, pp. 1251-1258, Sept. 2016.

[54] S. Y. Huang, and Y. H. Lee, "Tapered dual-plane compact electromagnetic bandgap microstrip filter structures," IEEE Trans. Microw. Theory Techn., vol. 53, no. 9, pp. 2656-2664, Sept. 2005.

[55] J. S. Lim, C. S. Kim, D. Ahn, Y. C. Jeong, and S. Nam, "Design of LowPass Filters Using Defected Ground Structure," IEEE Trans. Microw. Theory Techn., vol. 53, no. 8, pp. 2539-2545, Aug. 2005.

[56] C. Gao, Z. N. Chen, Y. Y. Wang, N. Yang, and X. M. Qing, "Study and suppression of ripples in passbands of series/parallel loaded EBG filters," IEEE Trans. Microw. Theory Techn., vol. 54, no. 4, pp. 1519-1526, June 2006.

[57] C. H. da Silva Santos, M. S. Goncalves, K. Claudio, A. C. Bordonalli, and H. E. H. Figueroa, "Bio-inspired algorithms and 2D Finite Element Method applied to Electromagnetic Band Gap structures design,"in 2009 3 rd European Conf. on Antennas and Propagation, Berlin, 2009, pp. 136140 .

[58] T. Yu-Bo, D. Yue, X. Zhi-Bin, S. Sha, and P. Tao, "Frequency characteristics of electromagnetic bandgap structure with bow-tie cells and its optimal design based on particle swarm optimization," IEEJ Trans. Electr. Electron. Eng., vol. 8, pp. 63, Nov. 2013.

[59] J. C. Myers, A. Kaur, K. Y. Park, and P. Chahal, "Design of thin-film quasi-optical terahertz components using evolutionary algorithms," 2015 IEEE 65th Electronic Components and Technology Conf. (ECTC), San Diego, CA, 2015, pp. 2012-2019.

[60] I. Arnedo et al., "Microwave periodic structures and synthesized structures with smooth profiles and their applications," 2016 IEEE MTT$S$ Latin America Microwave Conference (LAMC), Puerto Vallarta, 2016, pp. 1-3.

[61] T. Lopetegi, M. A. G. Laso, M. J. Erro, M. Sorolla, and M. Thumm, "Analysis and design of periodic structures for microstrip lines by using the coupled mode theory," IEEE Microw. Compon. Lett., vol. 12, no. 11, pp. 441-443, Nov. 2002.

[62] I. Arnedo, M. A. G. Laso, F. Falcone, D. Benito, and T. Lopetegi, "A Series Solution for the Single-Mode Synthesis Problem Based on the Coupled-Mode Theory," IEEE Trans. Microw. Theory Techn., vol. 56, no. 2, pp. 457-466, Feb. 2008.

[63] B. Z. Katsenelenbaum, L. Mercader, M. Pereyaslavets, M. Sorolla, and M. Thumm, Theory of Nonuniform Waveguides-the Cross-Section Method, London, UK: IEE Electromagnetic Waves Series, 44, 1998.

[64] I. Arnedo et al., "Analytical solution for the design of planar EBG structures with spurious-free frequency response," Microw. Opt. Technol. Lett., vol. 54, no. 4, pp. 956-960, Feb. 2012.

[65] J. de Dios Ruiz, F. L. Martinez, and J. Hinojosa, "Novel Compact WideBand EBG Structure Based on Tapered 1-D Koch Fractal Patterns," IEEE Antennas Wireless Propag. Lett., vol. 10, no., pp. 1104-1107, Oct. 2011.

[66] R. E. Collin, "Theory and Design of Wide-Band Multisection QuarterWave Transformers," Proc. IRE, vol. 43, no. 2, pp. 179-185, Feb. 1955.

[67] J. D. Rhodes, Theory of Electrical Filters. New York: Wiley, 1976.

[68] I. Arnedo, J. D. Schwartz, M. A. G. Laso, T. Lopetegi, D. V. Plant, and J. Azaña, "Passive Microwave Planar Circuits for Arbitrary UWB Pulse Shaping," IEEE Microw. Compon. Lett., vol. 18, no. 7, pp. 452-454, July 2008.

[69] M. Chudzik et al., "Design of transmission-type Nth-order differentiators in planar microwave technology," IEEE Trans. Microw. Theory Techn., vol. 60 , no. 11 , Nov. 2012. 
[70] M. Chudzik et al., "Novel synthesis technique for microwave circuits based on inverse scattering: Efficient algorithm implementation and application", Int. J. RF Microw. C. E., vol. 21, no. 2, pp. 164-173, Mar. 2011.

[71] I. Arnedo, I. Arregui, A. Lujambio, M. Chudzik, M.A.G. Laso, and T. Lopetegi, "Synthesis of Microwave Filters by Inverse Scattering Using a Closed-Form Expression Valid for Rational Frequency Responses", IEEE Trans. Microw. Theory Techn., vol. 60, no. 5, pp. 1244-1257, May 2012.

[72] R. Feced, M. N. Zerbas, and M. A. Muriel, "An Efficient Inverse Scattering Algorithm for the Design of Nonuniform Fiber Bragg Gratings," IEEE J. Quantum Electron., vol. 35, no. 8, pp. 1105-1115, Aug. 1999.

[73] R. G. Newton, "Inversion of reflection data for layered media - A review of Exact Methods," Geophys. J. Int., vol. 65, no. 1, pp. 191-215, Apr. 1981.

[74] J. Skaar, L. Wang, and T. Erdogan, "On the Synthesis of Fiber Bragg Gratings by Layer Peeling," IEEE J. Quantum Electron., vol. 37, no. 2, pp. 165-173, Feb. 2001.

[75] A. Papoulis, The Fourier Integral and its applications. New York. McGraw-Hill Electronic Science Series, 1962.

[76] R. E. Collin, Foundations for microwave engineering, Second Edition, New York, McGraw Hill, 1992.

[77] J. d. D. Ruiz, F. L. Martínez-Viviente, and J. Hinojosa, "Optimisation of chirped and tapered microstrip Koch fractal electromagnetic bandgap structures for improved low-pass filter design," IET Microwaves Antennas Propag., vol. 9, no. 9, pp. 889-897, June 2015.

[78] M. A. G. Laso et al., "Optimization of Tapered Bragg Reflectors in Microstrip Technology," Int. J. Infrared Millimeter Waves, Vol. 21, Issue 2, pp 231-245, Feb. 2000.

[79] H. Zhu, and J. Mao, "Miniaturized Tapered EBG Structure With Wide Stopband and Flat Passband," IEEE Antennas Wireless Propag. Lett., vol. 11, no. , pp. 314-317, Mar. 2012. 